\title{
Long-term survival of an elderly patient with advanced gastric cancer after combination therapy: a case report and literature review
}

\author{
Qingwei Li, Xuejun Xu, Dan Su, Tianshuo Zhou, Guangyu Wang and Zhiwei Li
}

\begin{abstract}
Background: Gastric cancer ranks the fifth most common cancer, and the third leading cause of cancer-related deaths worldwide. Gastric cancer with liver metastasis (GCLM) has devastating prognosis, however, optimal treatment of GCLM, especially in elderly patients, has yet to be clarified.

Case presentation: A 75-year-old man was diagnosed with advanced gastric cancer (GC), presenting with acute gastrointestinal bleeding and synchronous metastatic lesion in liver. Based on multidisciplinary team (MDT)'s decision, this patient underwent distal palliative gastrectomy with R1 margin. Histopathological diagnosis was stage IV gastric adenocarcinoma (pT3N2M1), HER2 negative. The patient was treated with chemotherapy and argonhelium cryoablation of liver and lung metastases.HER-2 gene amplification was identified in peripheral blood at later stage of therapy. The patient had been followed-up for 39 months, in sharp contrast to a median survival time of 13.8 months for majority of advanced GC.
\end{abstract}

Conclusions: Palliative distal gastrectomy in combination with chemotherapy and cryoablation significantly prolongs overall survival of an elderly patient with GCLM.

Keywords: Gastric cancer, Liver metastasis, Chemotherapy, Combination therapy

\section{Background}

Gastric carcinoma (GC) is the fourth most common malignancies and the third leading cause of cancer-related death worldwide, Surgical resection remains the only curative option [1]. In China, there are estimated 221,478 deaths per year due to GC, accounting for nearly half of the globally total deaths from GC [2]. Most of the patients with GC are diagnosed at advanced stages, frequently presenting invasion or metastasis [3]. Approximately, 25 and $30 \%$, respectively, of Chinese patients have early or late (metastatic) stage GC at diagnosis. While in the United States, $36 \%$ of patients have early stage at diagnosis [4].

At present, an optimal treatment of GCLM (which is classified as M1 clinical stage) remains debated [5, 6]. Hepatic metastases from GC are considered as

\footnotetext{
* Correspondence: Izhw0451@163.com

Department of Gastrointestinal Medical Oncology, Harbin Medical University Cancer Hospital, Harbin 150000, China

unresectable since these lesions present as multiple nodules, which are distributed in hepatic lobes, as well as extrahepatic organs $[7,8]$. Devastating prognosis is usually expected for unresectable GC. Under this circumstance, palliative chemotherapy will be highly recommended [9]. Among patients treated with chemotherapy alone, their 5 -year OS rate was only $1 \%$ (with a median survival time of 14 months). Conversion surgery may be attributed to long-term survival in selected patients.

The role of gastrectomy playing in treating metastatic GC (in the absence of urgent symptoms, such as bleeding or obstruction) is yet to be illustrated. A higher risk from surgery and longer recovery time are expected for elderly patients. Through reducing tumor volume, debulking surgery may prolong survival and/or delay the onset of life-threatening symptoms [10]. Elderly patients with GCLM are under-represented in clinical trials, with few reported studies in this setting. 
Here, we have described a specific case of long-term survival after palliative distal gastrectomy combined with chemotherapy and argon-helium cryoablation of liver and lung metastases. The observed improved outcome definitely merits a prospective study to explore potential survival benefits in specifically selected patients, especially for those who urgently require palliation of serious symptoms, such as bleeding or obstruction.

\section{Case presentation}

A 75-year-old man suffered from abdominal pain and melena. Emergency gastroscopy observed large curvature, posterior wall and small curvature of the antrum. Huge flat uplift occupying lesions were identified, with worm-like erosion edges, uneven bottom, visible bleeding from blood vessels and blood clots (Fig. 1 [green frame]; Fig. 2.a.b). An upholstery lesion (in the maximum diameter of $1.5 \mathrm{~cm}$ ) with white protrusions was observed close to the anterior wall of small curve. Whole body fluorine-18 fluorodeoxyglucose (18F-FDG) positron emission tomography/computed tomography (PET/ $\mathrm{CT}$ ) identified a hypodense mass in segment 6 of liver. Intense 18F-FDG distribution obtained a maximum standardized uptake value (SUV) of 3.5(Fig. 2, c-e). After MDT consultation, R0 resection might be impossible to achieve. Palliative chemotherapy was relatively contraindicated due to a high risk of gastrointestinal bleeding. The patient underwent palliative gastrectomy to prevent from bleeding and perforation. No liver metastatic lesion was resected. On microscopic examination, the primary tumor was identified as a well to mixed differentiated gastric adenocarcinoma (75\% papillary adenocarcinoma and $25 \%$ moderately differentiated tubular adenocarcinoma), which had invaded subserosa layer. Five of 35 lymph nodes were positive for metastases, without venous or lymphatic vasculature invasion. This GC tumor fulfilled the criteria of stage IVa (pT3N2M1), based on the American Joint Committee on Cancer (AJCC) TNM staging classification for carcinoma of the stomach (7th edition, 2012) [11]. This tumor was negative for HER2 amplification.

In the first month post-operation, this patient was transferred to our hospital for comprehensive evaluation. He had received 5 cycles of mFOLFOX6 (5-fluorouracil/folinic acid, oxaliplatin) regimen as the first-line chemotherapy. Liver metastatic lesions were shrunk, so that the efficacy was assessed as partial response (PR) (Fig. 3a). Then the patient received cryoablation with argon and helium on hepatic metastases; subsequently received post-operational chemotherapy (mFOLFOX6 protocol) for 3 cycles. The efficacy was evaluated as stable disease (SD) (Fig. 3b). Chemotherapy was stop ped due to grade3 neutropenia. The patient was regularly followed-up afterwards. Seven months after the cessation of treatment, CT scan identified new lesions in the lungs and liver (Fig. 1 [red frame]; Fig. 4a, b, c). The patient started to receive 3 cycles of Paclitaxel plus S-1 (second-line) chemotherapy since April 2015, however, the short-term effect was assessed as progressive disease (PD)(Fig. 4d, e).Then the patient received mFOLFOX6 chemotherapy for 7 cycles, with the efficacy defined as SD. Afterwards, pulmonary and hepatic metastatic lesions were treated with cryoablation separately (Fig. 5). The patient received another 3 cycles of

\section{Timeline}
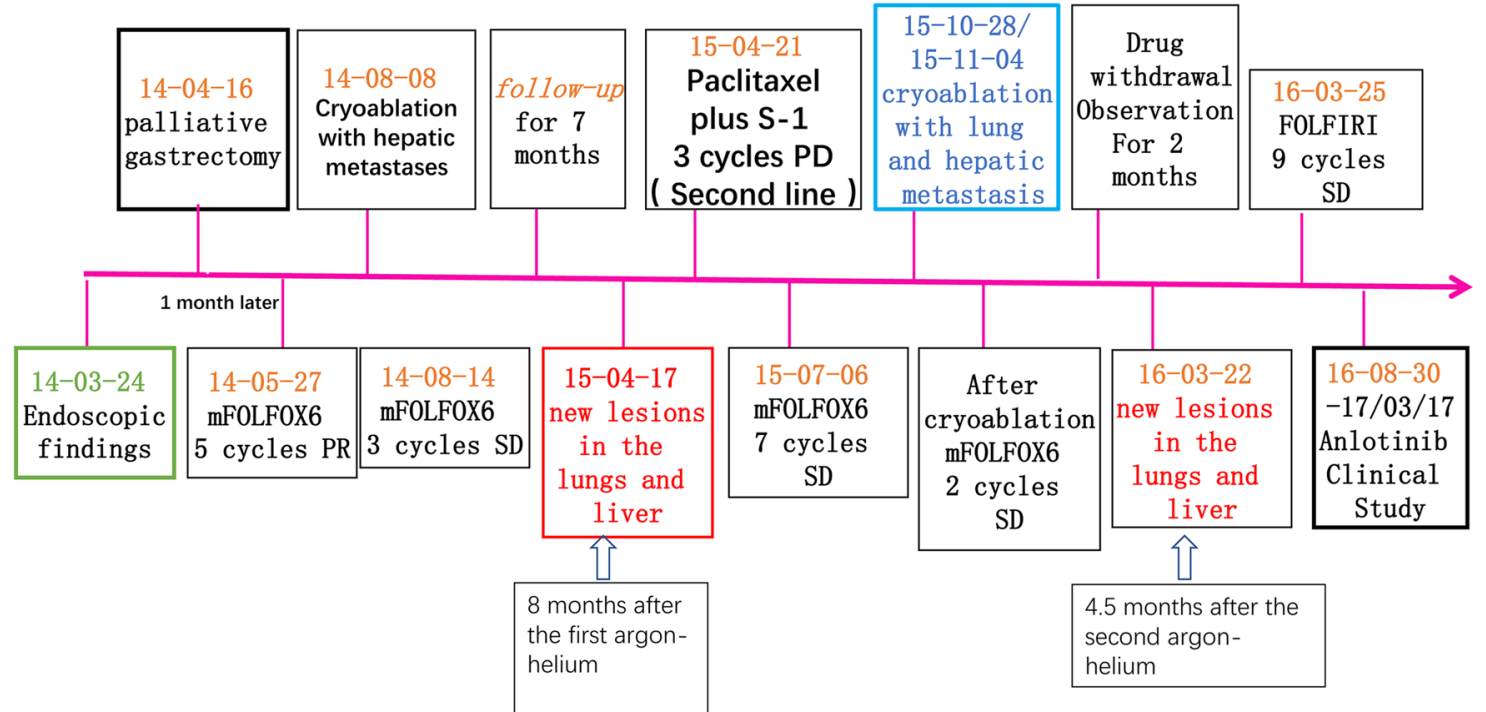

OS: 39 months

Fig. 1 Information of this case report has been organized into a timeline 

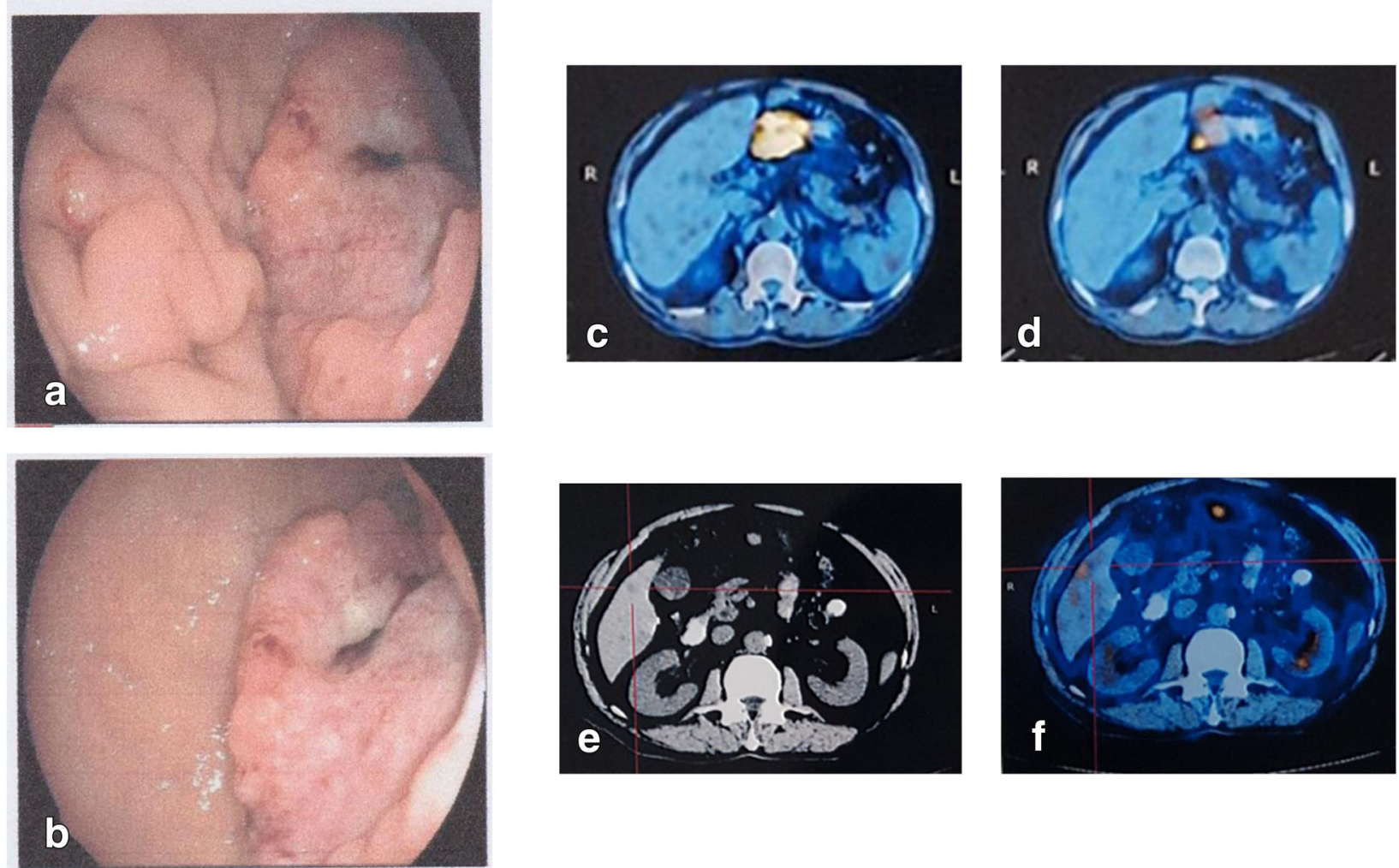

Fig. 2 Endoscopy and PET/CT revealed GCLM. a. posterior wall of the antrum: worm-like erosion edges, visible bleeding from blood vessels and blood clots. b. small curvature of the antrum, white protrusions. $\mathbf{c}$ The lesion was located in the gastric antrum(SUVmax13.3), indicating GC. $\mathbf{d}$ Lymph node metastasis was observed below the gastric antrum (SUVmax3.3). d. Computed Tomography Image revealed a single liver metastasis, located in segments 6 . e PET/CT identified localized radionuclide concentration (SUVmax3.5)

mFOLFOX6 regimen after cryoablation, undergoing regular observation. Six months later, new metastases were identified and the patient received FOLFIRI regimen chemotherapy for 9 cycles. The efficacy was evaluated as PD. At this point, HER-2 gene amplification was identified using peripheral blood sample (Fig. 6). During the entire treatment period, the patient's CA19-9 and CEA were basically maintained at normal levels (Additional file 1: Figure S1). On August 30, 2016, the patient was enrolled into a randomized, double-blind, placebo-controlled trial of Anlotinib, which was designed for advanced GC as second-line therapy. After taking 6 cycles of Anlotinib (12/10 mg, d1-14, qd, po, q3w), the efficacy was evaluated as SD (Fig. 7). Treatment-related proteinuria occurred during the second cycle. After the 8th cycle, the efficacy was evaluated as PD. The patient withdrew from the clinical trial on March 17, 2017. On July 24, 2017, the patient died and his overall survival time was 39 months.

\section{Discussion and conclusions}

GCLM refers to liver lesions originating from primary GC, which remains a major cause of GC-related deaths, with a 5 -year survival rate of $0-10 \%$ in unselected cases
[3]. Surgery with curative attempt is a key component for multidisciplinary approach. Hepatectomy may be performed on a small number of metastatic nodules, and not restricted to a solitary nodule, provided no other non-curable factors. Whether synchronous metastases have better prognosis than metachronous metastases remains controversial. Palliative gastrectomy could be considered for GCLM patients if they fulfill following criteria: (1) Through removing a bulky tumor, potential life-threatening symptoms such as obstruction, perforation, or bleeding maybe eliminated. (2) A decrease in tumor load may render the residual cancer cells more sensitive to adjuvant therapy. (3) Reduction in tumor volume may diminish nutrient burden on the patient exerted by the tumor. (4) As the tumor produce immunosuppressive cytokines, reducing the tumor burden may help activate anti-tumor immunologic machinery $[12,13]$. In this case, palliative gastrectomy contributes to prolonged survival.

Clinical benefit of resecting GCLM remains controversial, especially for elderly patients $[14,15]$. Clinical outcome for hepatic resection was disappointing due to high rates of recurrence and death. Occult intrahepatic 


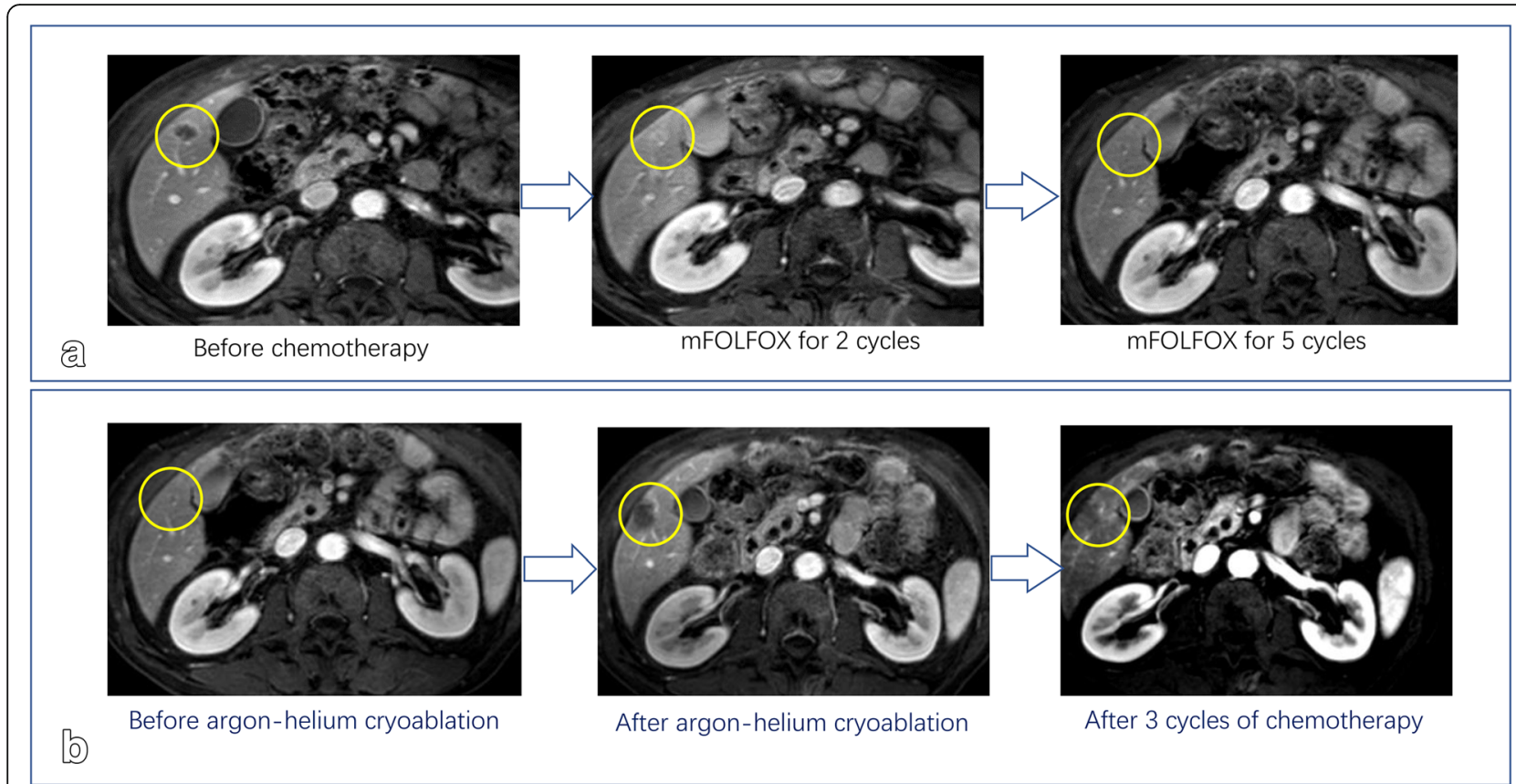

Fig. 3 Treatment protocol after reduction surgery. a The patient received 5 cycles of mFOLFOX6 regimen chemotherapy. The efficacy was assessed as PR. $\mathbf{b}$ The patient received cryoablation of argon and helium with hepatic metastases, and 3 cycles of mFOLFOX6 chemotherapy. The efficacy was evaluated as SD

metastases at the time of surgery may lead to high incidence of intrahepatic recurrence [16]. A second hepatic resection is rarely indicated. Postoperative monitoring of liver and adjuvant chemotherapy may become a feasible strategy for improving survival [17]. In this case, chemotherapy plays a critical role in improving prognosis. Hepatic lesions were used to screen drug sensitivity vs. resistance, and mFOLFOX regimen was indicated to be more effective. Local ablation has emerged as a promising alternative or complement to resection, especially for elderly patients with GCLM. Ablative techniques include radiofrequency thermal ablation (RFA) [18, 19], microwave ablation (MWA) [20, 21], and cryoablation [22]. The average survival time of ablative combined with chemotherapy for liver metastases was 16.1 months [23]. According to the retrospective study, the 5-year survival rate after ablative treatment was not significantly different from that of hepatectomy [24]. This patient underwent 3 times of local cryotherapy. After the first cryotherapy, perioperative chemotherapy was conducted. The patient's disease-free survival had reached 7 months. This procedure for elderly patients with GCLM is

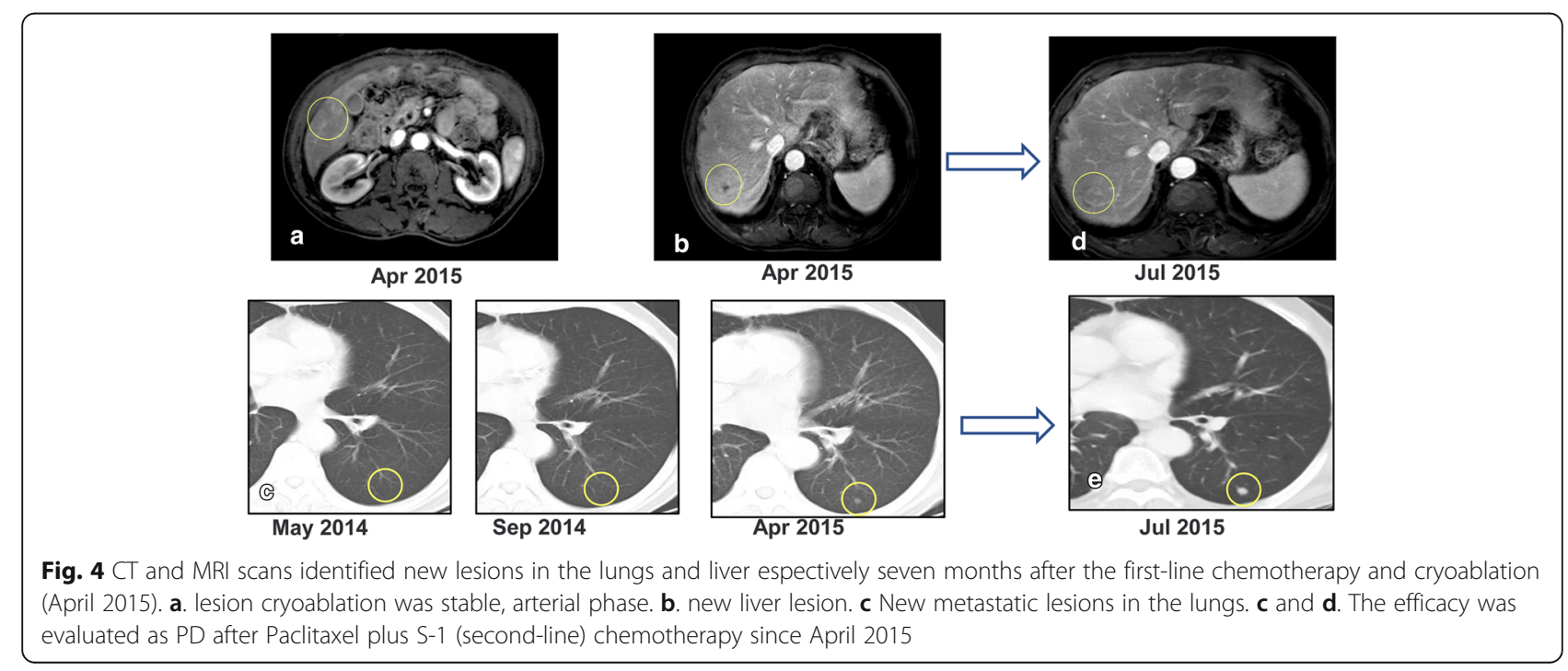




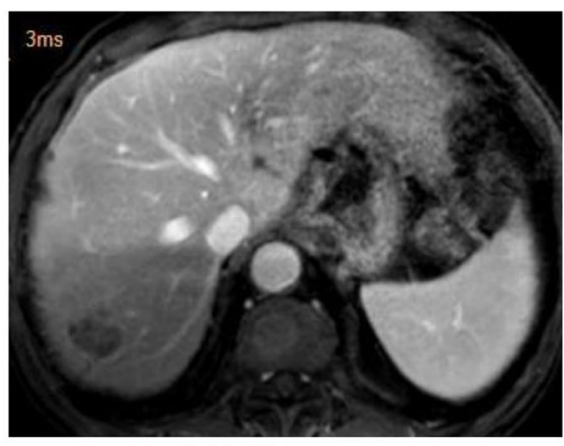

Oct 2015

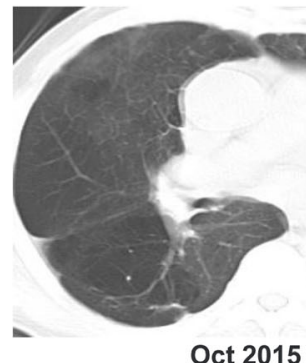

Before the cryoablation

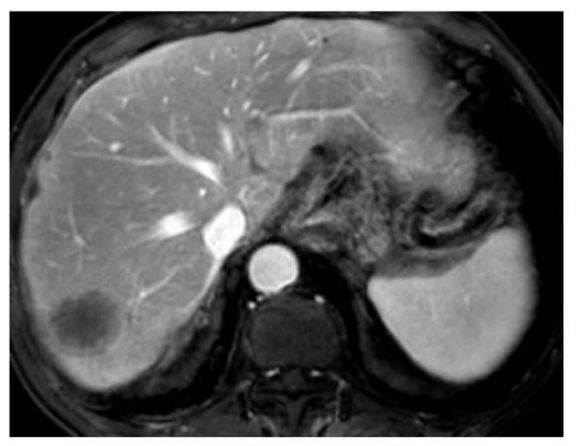

Jan 2016

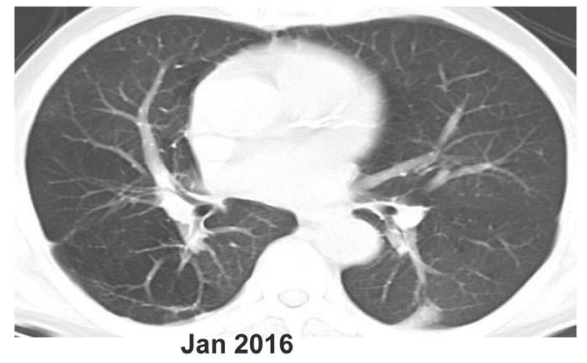

After the cryoablation

Fig. 5 Comparison of tumor status before and after the second cryotherapy

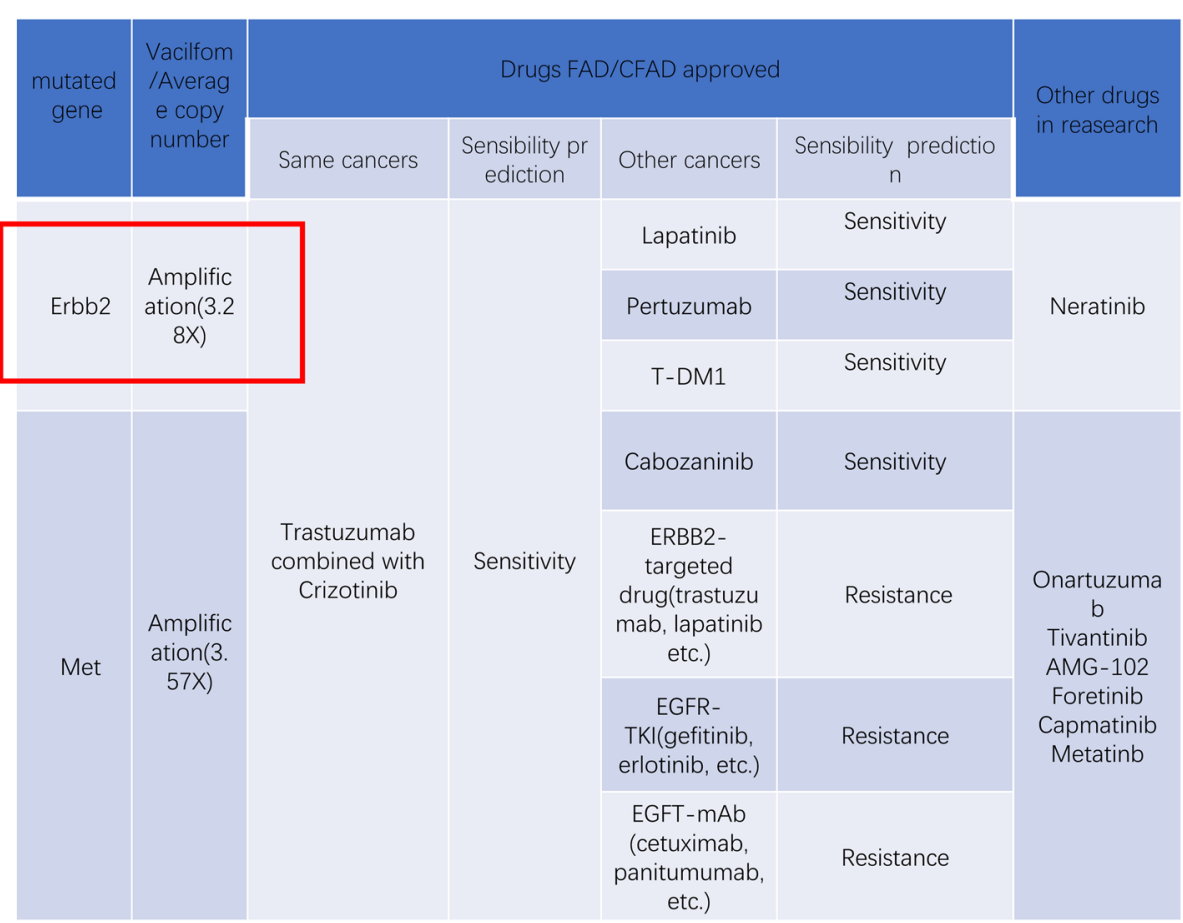

Fig. 6 Gene sequencing using peripheral blood sample.Red box highlighted HER2 amplification, which could be treated with pertuzumab (Herceptin) 

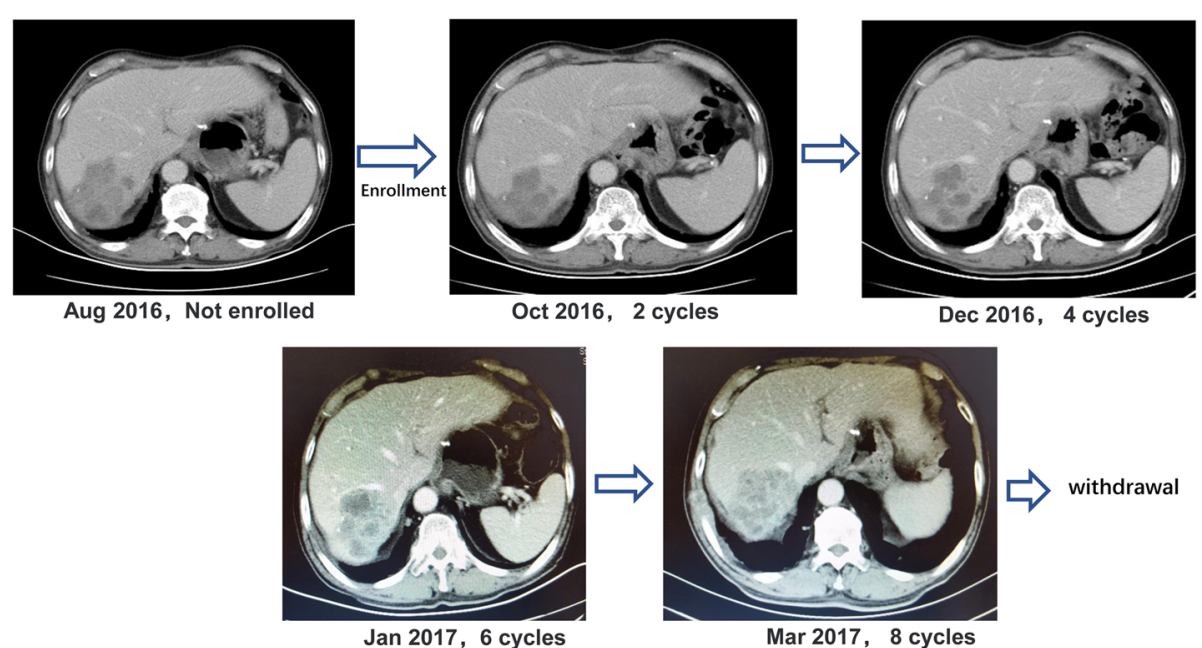

Fig. 7 The patient was enrolled into a randomized, double-blind, placebo-controlled clinical trial of Anlotinib

effective and safe (with low mobility), which may be performed repeatedly on an outpatient basis with a good palliative effect.

Supporting evidence from clinical trials is required to guide decision-making when treating elderly patients with GCLM. Age alone is no contraindication for resections of GI. Although no survival benefit for neoadjuvant treatment in patients over 70 years is found [25]. In this case, if the patient was not at risk of bleeding, we may prefer neoadjuvant chemotherapy. Preoperative chemotherapy significantly prolongs the survival of the esophago-gastric adenocarcinomas patient with primary resection [26]. In regards to chemotherapy for advanced/metastatic GC, the experience from general population is unlikely to be directly applicable to elderly patients [27]. It is inappropriate to estimate tolerability of elderly patients to chemotherapy based only on chronological age without considering functions of major organs, comorbidities and medical history. However, an ideal index to comprehensively assess vulnerability of aged individual has yet to be established. Therefore, more comprehensive clinical studies with larger sample sizes on elderly patients with GCLM are guaranteed to validate our findings in the near future.

This patient participated in a clinical study, which was helpful to extend overall survival. Anlotinib is an oral formulation of a small molecule inhibitor of multiple receptor tyrosine kinases, with abroad spectrum of inhibitory effects on tumor growth and angiogenesis. Anlotinib has been approved in China for treatment of patients with locally advanced or metastatic non-small cell lung cancer (NSCLC), who have undergone progression or recurrence after $\geq 2$ lines of systemic chemotherapy. The efficacy of Anlotinib in patients with stage 3B/4 NSCLC or metastatic renal cancer (mRCC) has been demonstrated. During 6 cycles of enrollment into this clinical trial, the efficacy was evaluated as SD.

This patient was HER-2 negative based on postoperative pathological examination. However, in the latter stage of treatment, genetic test using peripheral blood identified amplification in HER-2 gene. On one hand, loss of HER2-positive status occurs after neoadjuvant therapy in patients with primary HER2-positive breast cancer [28]. On the other hand, 3.4\% of breast cancer patients with HER2-negative tumors before chemotherapy changed to HER2-positive afterwards [29]. According to this case, we guess that the alteration in HER2 expression may happen in $\mathrm{GC}$, which may be resulted from resistance to chemotherapy as HER2 amplification means poor prognosis.

For elderly patients with GCLM, combination therapy has efficacy. MDT consultation facilitates the evaluation of clinical stage, feasibility, risk and benefit of individual treatment modality. Palliative gastrectomy for GCLM is reasonable and safe; however, the patients must be strictly selected. Systemic chemotherapy combined with local cryoablation is an important choice for GCLM. To participate inappropriate clinical trials may be indispensable.

\section{Additional file}

Additional file 1: Figure S1. Levels of change in ca 19-9 and CEA during patient treatment. During the entire treatment period, the patient's CA19-9 and CEA were basically maintained at normal levels. A.CA19-9; B. CEA. (TIF $27264 \mathrm{~kb}$ )

\section{Abbreviations}

18F-FDG: fluorine-18 fluorodeoxyglucose; CT scan: computed tomography scan; FOLFIRI: fluorouracil, leucovorin, and irinotecan; GC: gastric cancer; GCLM: gastric cancer with liver metastasis; HER-2: Receptor tyrosine-protein 
kinase erbB-2; MDT: multidisciplinary team; mFOLFOX6: Modified Fluorouracil, Leucovorin, and Oxaliplatin; MWA: microwave ablation; OS: overall survival; PD: progression disease; PET/CT: positron emission tomography/computed tomography; PR: partial remission; RFA: radiofrequency thermal ablation; SD: stable disease; SUV: standardized uptake value

\section{Acknowledgements}

Not Applicable.

\section{Funding}

This work was supported by the National Natural Science Foundation of China(Grand No.81773210)and Haiyan Fund of Harbin medical University (Grand No. JJLX2016-01).The funding body had no role in the design of the study and collection, analysis, and interpretation of data and in writing this manuscript.

\section{Availability of data and materials}

The datasets used and/or analyzed during the current study are available from the corresponding authors upon reasonable request.

\section{Authors' contributions}

Contributed substantially to study conception and design, data acquisition, data analysis and interpretation: QWL, XJX, DS, TSZ, GYW, ZWL. Involved in drafting the manuscript or revising it critically: QWL, XJX, ZWL. Gave final approval of the version to be published: QWL, XJX, DS, TSZ, GYW, ZWL. Agreed to be held accountable: ZWL. All authors read and approved the final manuscript.

\section{Ethics approval and consent to participate}

As it is a case report, ethics approval is not applicable. The patient was enrolled in a clinical trial, which is approved by the Ethics Committee of Tumor Hospital of Harbin Medical University (Ethical code:15-12).

\section{Consent for publication}

Consent to publish the case and all details and images described was obtained from the individual in this case report.

\section{Competing interests}

The authors declare that they have no competing interests. The patient permitted the publication of the case, the clinical details and images.

\section{Publisher's Note}

Springer Nature remains neutral with regard to jurisdictional claims in published maps and institutional affiliations.

\section{Received: 23 October 2018 Accepted: 8 May 2019}

\section{Published online: 16 May 2019}

\section{References}

1. Thun MJ, DeLancey JO, Center MM, Jemal A, Ward EM. The global burden of cancer: priorities for prevention. Carcinogenesis. 2010;31(1):100-10.

2. World Health Organization (2014) GLOBOCAN2012: Estimated Cancer Incidence MaPWi.

3. Liu J, Chen L. Current status and progress in gastric cancer with liver metastasis. Chin Med J. 2011;124(3):445-56.

4. Strong VE, Wu AW, Selby LV, Gonen M, Hsu M, Song KY, Park CH, Coit DG, Ji JF, Brennan MF. Differences in gastric cancer survival between the U.S. and China. J Surg Oncol. 2015;112(1):31-7.

5. Vigano L, Vellone M, Ferrero A, Giuliante F, Nuzzo G, Capussotti L. Liver resection for gastric cancer metastases. Hepato-gastroenterology. 2013; 60(123):557-62.

6. Hwang SE, Yang DH, Kim CY. Prognostic factors for survival in patients with hepatic recurrence after curative resection of gastric cancer. World I Surg. 2009;33(7):1468-72.

7. Sakamoto Y, Sano T, Shimada K, Esaki M, Saka M, Fukagawa T, Katai H, Kosuge T, Sasako M. Favorable indications for hepatectomy in patients with liver metastasis from gastric cancer. J Surg Oncol. 2007;95(7):534-9.

8. Takemura N, Saiura A, Koga R, Arita J, Yoshioka R, Ono Y, Hiki N, Sano T, Yamamoto J, Kokudo $\mathrm{N}$, et al. Long-term outcomes after surgical resection for gastric cancer liver metastasis: an analysis of 64 macroscopically complete resections. Langenbeck's Arch Surg. 2012;397(6):951-7.
9. Fukuchi M, Ishiguro T, Ogata K, Suzuki O, Kumagai Y, Ishibashi K, Ishida H, Kuwano H, Mochiki E. Prognostic role of conversion surgery for Unresectable gastric Cancer. Ann Surg Oncol. 2015;22(11):3618-24.

10. Fujitani K, Yang HK, Mizusawa J, Kim YW, Terashima M, Han SU, Iwasaki Y, Hyung WJ, Takagane A, Park DJ, et al. Gastrectomy plus chemotherapy versus chemotherapy alone for advanced gastric cancer with a single noncurable factor (REGATTA): a phase 3, randomised controlled trial. Lancet Oncol. 2016;17(3):309-18.

11. Edge SB, Compton CC. The American joint committee on Cancer: the 7th edition of the AJCC Cancer staging manual and the future of TNM. Ann Surg Oncol. 2010;17(6):1471-4.

12. McCarter MD, Fong Y. Role for surgical cytoreduction in multimodality treatments for cancer. Ann Surg Oncol. 2001;8(1):38-43.

13. Saidi RF, ReMine SG, Dudrick PS, Hanna NN. Is there a role for palliative gastrectomy in patients with stage IV gastric cancer? World J Surg. 2006; 30(1):21-7.

14. Cheon S, Rha S, Jeung H-C, Im C-K, Kim S, Kim H, Ahn J, Roh J, Noh S, Chung $\mathrm{H}$. Survival benefit of combined curative resection of the stomach (D2 resection) and liver in gastric cancer patients with liver metastases. Ann Oncol. 2008;19(6):1146-53.

15. Sakamoto Y, Ohyama S, Yamamoto J, Yamada K, Seki M, Ohta K-i, Kokudo N, Yamaguchi T, Muto T, Makuuchi M. Surgical resection of liver metastases of gastric cancer: an analysis of a 17-year experience with 22 patients. Surgery. 2003;133(5):507-11.

16. Nomura T, Kamio Y, Takasu N, Moriya T, Takeshita A, Mizutani M, Hachiya O, Hirai I, Kimura W. Intrahepatic micrometastases around liver metastases from gastric cancer. J Hepato-Biliary-Pancreat Surg. 2009;16(4):493-501.

17. Kakeji Y, Morita M, Maehara Y. Strategies for treating liver metastasis from gastric cancer. Surg Today. 2010;40(4):287-94.

18. Kim HR, Ha Cheon S, Lee K-H, Ryun Ahn J, Jeung H-C, Sook Lee S, Cheol Chung $\mathrm{H}$, Hoon Noh S, Young Rha S. Efficacy and feasibility of radiofrequency ablation for liver metastases from gastric adenocarcinoma. Int J Hyperth. 2010:26(4):305-15.

19. Kim HO, Hwang SI, Hong HP, Yoo CH. Radiofrequency ablation for metachronous hepatic metastases from gastric cancer. Surg Laparosc Endosc Percutan Tech. 2009;19(3):208-12.

20. Boutros C, Somasundar P, Garrean S, Saied A, Espat N. Microwave coagulation therapy for hepatic tumors: review of the literature and critical analysis. Surg Oncol. 2010;19(1):e22-32.

21. Yu J, Liang P, Yu X, Liu F, Chen L, Wang Y. A comparison of microwave ablation and bipolar radiofrequency ablation both with an internally cooled probe: results in ex vivo and in vivo porcine livers. Eur J Radiol. 2011;79(1): 124-30.

22. Mayo SC, Pawlik TM. Thermal ablative therapies for secondary hepatic malignancies. Cancer J. 2010;16(2):111-7.

23. Chen J, Tang Z, Dong X, Gao S, Fang H, Wu D, Xiang D, Zhang S. Radiofrequency ablation for liver metastasis from gastric cancer. Eur J Surg Oncol. 2013:39(7):701-6.

24. Guner A, Son T, Cho I, Kwon IG, An JY, Kim HI, Cheong JH, Noh SH, Hyung WJ. Liver-directed treatments for liver metastasis from gastric adenocarcinoma: comparison between liver resection and radiofrequency ablation. Gastric Cancer. 2016;19(3):951-60.

25. Nienhueser H, Kunzmann R, Sisic L, Blank S, Strowitzk MJ, Bruckner T, Jage D, Weichert W, Ulrich A, Buchler MW, et al. Surgery of gastric cancer and esophageal cancer: does age matter? J Surg Oncol. 2015;112(4):387-95.

26. Schmidt T, Alldinger I, Blank S, Klose J, Springfeld C, Dreikhausen L, Weichert W, Grenacher L, Bruckner T, Lordick F, et al. Surgery in oesophago-gastric cancer with metastatic disease: treatment, prognosis and preoperative patient selection. Eur J Surg Oncol. 2015;41(10):1340-7.

27. Association JGC. Japanese gastric cancer treatment guidelines 2014 (ver. 4). Gastric Cancer. 2017:20(1):1-19.

28. De La Cruz LM, Harhay MO, Zhang P, Ugras S. Impact of neoadjuvant chemotherapy on breast Cancer subtype: does subtype change and, if so, how? : IHC profile and neoadjuvant chemotherapy. Ann Surg Oncol. 2018; 25(12):3535-40.

29. Niikura N, Tomotaki A, Miyata H, Iwamoto T, Kawai M, Anan K, Hayashi N, Aogi $\mathrm{K}$, Ishida T, Masuoka $\mathrm{H}$, et al. Changes in tumor expression of HER2 and hormone receptors status after neoadjuvant chemotherapy in 21,755 patients from the Japanese breast cancer registry. Ann Oncol. 2016;27(3): $480-7$. 